\title{
Biomarker validity in the critically ill: all must face the (continuous) renal replacement challenge!
}

\author{
Patrick M. Honore*, Rita Jacobs, Inne Hendrickx, Elisabeth De Waele, Viola Van Gorp and Herbert D. Spapen \\ See related research by Roderburg et al., http://www.ccforum.com/content/19/1/271
}

We embrace with enthusiasm the recent study by Roderburg et al. [1] identifying osteopontin (OPN) as a valuable early prognostic biomarker in critically ill patients. Of note is that these investigators did not provide details on the incidence of acute kidney injury or the need for renal replacement therapy (RRT) in their patient population.

We recently argued that currently used markers of systemic inflammation such as $\mathrm{C}$-reactive protein and procalcitonin are substantially cleared during continuous renal replacement therapy (CRRT) and thus may lose sensitivity to evaluate the degree and evolution of inflammation [2]. OPN is a highly negatively charged protein. Its nascent molecular weight (MW) approximates $32 \mathrm{kDa}$ with slight variations due to post-translational modification or proteolytic cleavage [3]. OPN is not eliminated by slow extended dialysis [4]. This is not surprising, however, because the $5 \mathrm{kDa}$ membrane cutoff point for molecular diffusion with this technique lies largely below the MW of OPN. Theoretically, continuous hemofiltration may remove OPN from the circulation but evidence is lacking. Moreover, CRRT is increasingly performed with novel membranes such as the surface-treated acrylonitrile 69 membrane [5]. Surface treatment implies coating with a polyethylene imine biopolymer resulting in a more neutral membrane surface composed of areas with a high density of positive charges. In addition to being highly biocompatible and permeable, this membrane displays potent adsorptive capacity.

More information regarding an eventual significant "loss" of OPN by filtration and/or membrane adsorption during CRRT is imperative before this protein can be accepted as a valid prognostic biomarker.

\section{Authors' response: impact of renal replacement therapy on osteopontin levels in critically ill patients Christoph Roderburg, Fabian Benz, Hans-Joerg Hippe, Tom Luedde, Christian Trautwein, Norbert Frey, Alexander Koch, Frank Tacke and Mark Luedde}

We are grateful for the letter from Honore et al. raising the important question of an eventual "loss" of OPN by filtration during (C)RRT in critically ill patients. We cannot fully exclude this bias but we have performed additional analyses on our cohort of critically ill medical patients [1], suggesting that OPN serum levels at day 3 after admission to the ICU remain a valuable prognostic biomarker in critically ill patients. Although the records from our database only reveal whether patients received RRT or not (and, unfortunately, not the exact modalities, time points, and durations), OPN levels at day 3 were significantly higher in patients receiving RRT compared with patients without RRT (Fig. 1a), arguing against a

\footnotetext{
* Correspondence: Patrick.Honore@az.vub.ac.be

ICU Department, Universitair Ziekenhuis Brussel, Vrije Universiteit Brussel,

101 Laarbeeklaan, 1090 Jette, Brussels, Belgium
} 

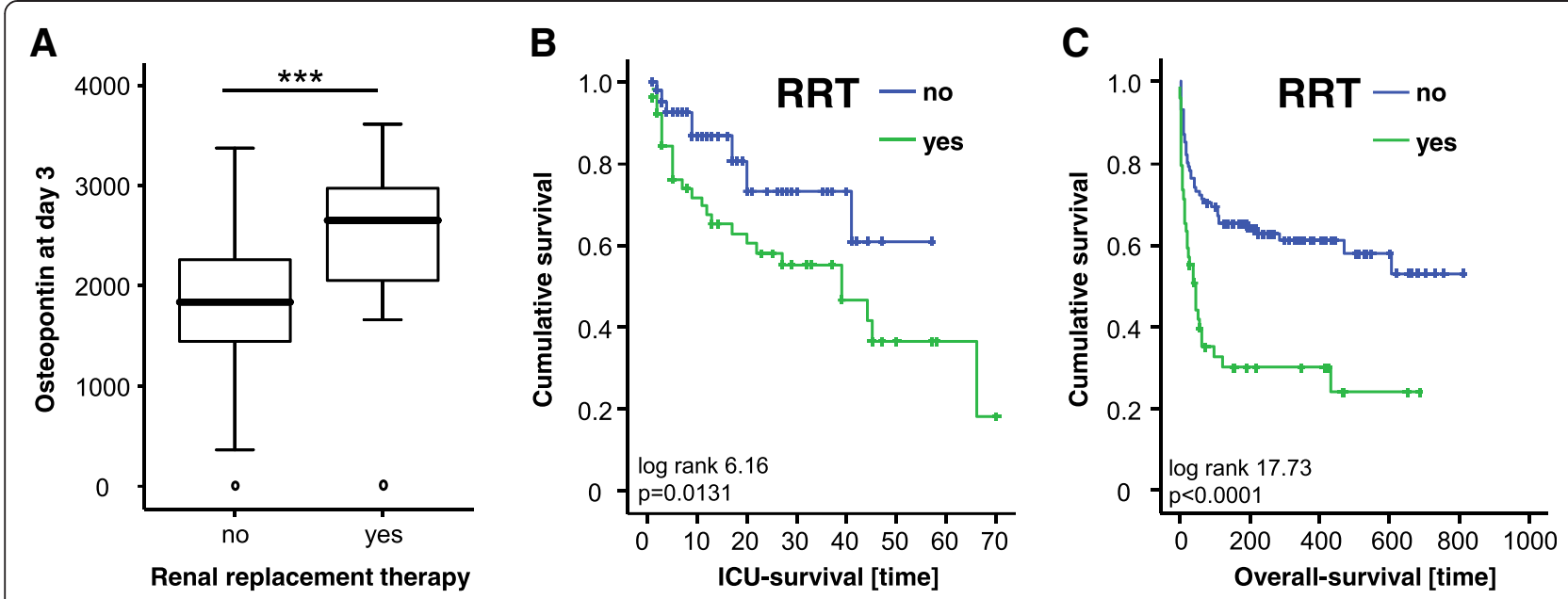

Fig. 1 OPN serum levels are elevated in critically ill patients with RRT. a OPN serum concentrations at day 3 of medical ICU treatment were significantly higher $(P<0.001, U$ test) in patients that received RRT $(n=34)$ compared with patients without need for this therapy $(>n=78)$. b, c Patients with a need for RRT displayed a significantly impaired ICU survival and overall survival in comparison with other patients, based on Kaplan-Meier curve analysis. ${ }^{* * *} P<0.001$. RRT Renal replacement therapy

significant loss due to filtration. In line with the prognostic role of OPN levels in critically ill patients, need for RRT (and thus presence of higher OPN levels) was associated with a dramatically impaired survival (Fig. 1b, c). Moreover, multivariate analysis including RRT as a variable revealed that OPN levels remained an independent prognostic marker in critically ill patients, as reported previously in our study [1]. Finally, OPN concentrations were strongly correlated to procalcitonin serum levels, which have been demonstrated to be independent of RRT in critical illness [6].

Taken together, our results do not support the concern that OPN serum levels might not be a valid prognostic parameter in patients with acute kidney injury undergoing RRT. Nevertheless, we cordially thank Honore et al. for their valuable comment on our article, as further prospective trials considering renal failure and replacement should be conducted to properly address this question.

\section{Abbreviations}

CRRT: continuous renal replacement therapy; MW: molecular weight; OPN: osteopontin; RRT: renal replacement therapy.

\section{Competing interests}

The authors declare that they have no competing interests.

\section{Authors' contributions}

PMH and HDS designed the article. PMH, R, $I H, E D W, W G$, and HDS participated in drafting the manuscript. All authors read and approved the manuscript.

Published online: 06 December 2015

\section{References}

1. Roderburg C, Benz F, Cardenas DV, Lutz M, Hippe HJ, Luedde T, et al. Persistently elevated osteopontin serum levels predict mortality in critically ill patients. Crit Care. 2015;19:271.

2. Honore PM, Jacobs R, Hendrickx I, De Waele E, Van Gorp V, Spapen HD. "Biomarking" infection during continuous renal replacement therapy: still relevant? Crit Care. 2015:19:232.

3. Subraman V, Thiyagarajan M, Malathi N, Rajan ST. OPN—revisited. J Clin Diagn Res. 2015;6:10-3.

4. Lorensen JM, Hafer C, Faulhaber-Walter R, Kumpers PR, Kielstein J, Haller $\mathrm{H}_{4}$ et al. Osteopontin predicts survival in critically ill patients with acute kidney injury. Nephrol Dial Transplant. 2011;26:531-7.

5. Honore PM, Jacobs R, Joannes-Boyau O, De Regt J, De Waele E, van Gorp V, et al. Newly designed CRRT membranes for sepsis and SIRS - a pragmatic approach for bedside intensivists summarizing the more recent advances: a systematic structured review. ASAIO J. 2013:59:99-106.

6. Meisner M, Huttemann E, Lohs T, Kasakov L, Reinhart K. Plasma concentrations and clearance of procalcitonin during continuous venovenous hemofiltration in septic patients. Shock. 2001;15:171-5. 